\title{
MS060.P01
}

\section{Exploring liquid-liquid' transitions in ZnSe at extreme conditions}

Sakura Pascarelli ${ }^{1}$, Can Yildirim² ${ }^{2}$ Jean-Yves Raty ${ }^{3}$, Innokenty Kantor ${ }^{2}$, Raffaella Torchio ${ }^{2}$, Angelika Dorothea Rosa ${ }^{2}$, Gaston Garbarino ${ }^{2}$, Gunnar Weck ${ }^{4}$, Jean-Pierre Gaspard ${ }^{3}$

${ }^{1}$ European Synchrotron Radiation Facility, Grenoble, France, ${ }^{2}$ Esrf, Grenoble, France, ${ }^{3} 1$ SPIN-Université de Liège, Institut de Physique B5, 4000 Sart-Tilman, Belgium, ${ }^{4}$ CEA, Paris, France

E-mail: sakura@esrf.fr

Here, we report on the fingerprints of 4-fold to 6-fold transition in liquid ZnSe at extreme pressure and temperature conditions up to $42 \mathrm{GPa}$ and more than $3000 \mathrm{~K}$ using X-ray absorption spectroscopy (XAS) combined with laser-heated diamond anvil cell techniques and complementary ab initio molecular dynamics simulations. Among pressure induced structural modifications, liquid-liquid' ( $L-L^{\prime}$ ) transitions have attracted much interest both experimentally and theoretically due to their peculiarity. Unlike most $L-L^{\prime}$ transitions that only show slight modifications of the bond distances between atoms (i.e., $\mathrm{P}$, Na, supercooled Si) $[1,2,3]$, strong $L-L^{\prime}$ transitions have been theoretically predicted for a few II-VI semiconductor compounds such as ZnSe, CdSe and CdTe, given the following two conditions are satisfied: Firstly, the melting of the sp3 bonded phase (4-fold coordination) at ambient pressure results in a liquid structure that remains approximately 4-fold coordinated; and secondly, the presence of a semiconductor to metal phase transition under pressure [4, 5, 6, 7]. Our results show that solid and liquid $Z n S e$ undergoes a series of structural modifications at various $P, T$ values that satisfy the conditions above. The red shift in $\mathrm{Zn} \mathrm{K}$ edge energy observed at around $7 \mathrm{GPa}$ upon increasing temperature suggests a metallization event before melting to a possible 6-fold coordinated liquid structure. These findings are supported by our simulation results showing a pronounced difference in the first diffraction peak of the calculated structure factor at high pressures indicating a 4-fold to 6-fold coordination change in liquid ZnSe. Our results may provide additional insight for such transitions that may be observed for similar tetrahedrally coordinated II-VI systems.

[1] Raty, J.Y, Eric S., and Stanimir A. B. Nature 449.7161 (2007): 448-451.

[2] Katayama, Yoshinori, et al. Nature 403.6766 (2000) 170-173.

[3] Ganesh, P., and M. Widom. Physical review letters 102.7 (2009): 075701.

[4] T. Narushima et al., Joint 20th AIRAPT-43th EHPRG Proceedings (2005),

[5] T. Tsukatani, A. Chiba, K. Tsuji, Journal of Physics: Conference Series (2010): 215012076.

[6] J.-P. Gaspard, J.-Y. Raty, R. Céolin and R. Bellissent, J. non Cryst. Solids, (1996): 205-207, 75-78

[7] Hattori, T., Kinoshita, T., Narushima, T., Tsuji, K., \& Katayama, Y. (2006). Physical Review B, $73(5), 054203$.

Keywords: Liquide-liquide phase transitions, laser-heated DAC, XAS 